Leib - Körper - Maschine.

Zum Problem der leiblichen Aneignung technischer Artefakte

Reinhold Esterbauer

Einleitung: „Body Art“ und „Body Modification”

In der Kunst ist der menschliche Körper immer schon Thema gewesen. Seit der Aktionskunst - etwa im Wiener Aktionismus bei Günter Brus oder bei Valie Export - wurde das Augenmerk aber in veränderter und intensivierter Form auf den Körper gerichtet. Mittlerweile gibt es verschiedene Ausprägungen von Body Art und Carnal Art sowie Versuche, den Körper in Szene zu setzen und zu modellieren. Eine extreme Variante bildet die provokative Praxis, den eigenen Körper nicht nur tätowieren oder mit Metallringen an allen möglichen Stellen „tunen“, sondern auch operativ verformen und durch künstliche Implantate verändern zu lassen. ${ }^{1}$ Solche Body Modification legt es darauf an, den eigenen Körper oder das eigene „Fleisch“ zum Kunstmaterial zu machen und Artefakte unter die Haut einpflanzen zu lassen, um damit den eigenen Körper umzugestalten. So lassen sich Menschen am Kopf oder an den Schultern Hörner implantieren, Zungen spalten, Magnete unter die Fingerspitzen operieren oder an Computerteile erinnernde Implantate unter der Haut der Handoberfläche anbringen.

Was mittels Aktionskunst, Carnal Art oder Body Modification auch ins Bewusstsein gerückt wurde und wird, ist die Frage, inwieweit es möglich ist, Teile des menschlichen Körpers durch andere zu ersetzen. Diese Frage ist jenseits körperlicher Inszenierung auch ein medizinisches Problem. Stand mit der Organtransplantation zunächst das medizinische und psychologische Thema zur Diskussion, inwieweit fremde Organe in einen anderen Körper implantiert werden können, so stellt sich heute die Frage, wie viele fremde Organe ein Körper integrieren kann, wenn die Person, um deren Körper es geht, nicht nur am Leben, sondern auch sie selbst bleiben soll. Dazu kommt die Frage der Prothetik, also das Problem, inwie-

1 Bekannt geworden dafür sind etwa die französische Künstlerin ORLAN, die Schweizer Body-Artistin Sandra Caracciolo oder María José Cristerna, die als die meist verformte Körperkünstlerin gilt. 
weit technisch hergestellte Gliedmaßen oder künstliche Organe Teil eines Menschen werden können, wenn sie Funktionen kranker oder amputierter Körperteile übernehmen sollen. Das führt schließlich zur Frage, ob denn nicht der ganze Körper einmal durch eine Maschine ersetzt werden könne, man also den Menschen künstlich herstellen oder durch einen Roboter zu erneuern vermag, ohne dass sich dadurch die Identität der Person veränderte.

Ich möchte mich im Folgenden auf das Problem beschränken, wie technische Artefakte als Teil des Körpers angeeignet werden können und welche leib-körperlichen Grundbedingungen dafür verantwortlich sind, dass eine partielle Aneignung möglich ist, andere Formen aber scheitern. Dazu ist es freilich notwendig, zunächst die leib-körperliche Struktur des Menschen in den Blick zu nehmen, bevor auf die leibliche Integration technischer Artefakte eingegangen werden kann.

\section{Der Leibkörper}

Die Erfahrung des eigenen Leibes bzw. Körpers erweist sich als zwiespältig. Auf der einen Seite ist es möglich, zu seinem Körper in Distanz zu treten, wenn man etwa Körperpflege betreibt oder sein Gewicht kontrolliert. Besonders wenn es darum geht, dass andere sich am eigenen Körper zu schaffen machen - z. B. beim Frisör oder bei einer medizinischen Behandlung -, gelangt der Körper in die Position des Gegenübers, die es erlaubt, den fremden, aber auch den eigenen Körper zu objektivieren, ihn beispielsweise zu betrachten, zu vermessen, zu analysieren oder invasiv zu verändern. Auf der anderen Seite zeigt sich der Körper bzw. Leib zugleich als Ausdrucksmedium, das es einem erlaubt, sich in der Welt zu vollziehen und sowohl räumlich als auch zeitlich in der Welt zu leben. Nur mittels des eigenen Leibes ist man hier und jetzt präsent und ist einem die Möglichkeit eröffnet, die eigene Umgebung wahrzunehmen und in sie hineinzuwirken. Selbst das Hantieren am eigenen Körper ist wieder nur leiblich möglich, z.B. wenn man sich die Haare kämmt oder die Zähne putzt.

Im Deutschen ist es möglich, diese zwei Bereiche mit separaten Begriffen zu bezeichnen. So meint der Ausdruck „Körper“ die Dimension, in der man sich gegenübertreten kann, der Begriff „Leib“ hingegen jene, worin man sich ausdrückt und sich selbst vollzieht. Helmuth Plessner hat, um die Unterschiede der Verhältnisse, die man zum eigenen Körper und zum eigenen Leib hat, hervorzuheben, festgehalten, dass man seinen Körper habe, sein Leib aber sei. So schreibt er: „Ein Mensch ist immer zugleich Leib (Kopf, Rumpf, Extremitäten mit allem, was darin ist) [...] und hat diesen 
Leib als diesen Körper.“ (Plessner 1982, 238; Hervorh. im Original; vgl. Fuchs 2013, 82-84)

$\mathrm{Zu}$ beachten ist, dass der Körper, den ich habe, und der Leib, der ich bin, nicht zwei getrennte Bereiche sind, sondern vielmehr zwei Dimensionen ein und desselben meinen. Es treten beide Seiten in verschiedenen Situationen in unterschiedlicher Intensität hervor, ohne dass der jeweils andere Bereich völlig verschwinden würde. So kann man sagen: Mein Körper ist der Leib, den ich habe, und mein Leib ist der Körper, der ich bin. Die Verknüpfung von Körper und Leib lässt sich also nicht auflösen. Vielmehr kann man von einer „Polarität“ sprechen, „in der wir uns ständig zwischen dem Leib-Sein und dem Körper-Haben hin- und herbewegen". (Fuchs 2013, 84; Hervorh. im Original) Anders als Hermann Schmitz meint, ${ }^{2}$ ist trotz der Polarität von Körper und Leib an deren untrennbarer Verknüpfung festzuhalten, ist doch der Leib nicht ohne Körper zu haben und ist ein Körper ohne Leib ein bloß toter Körper. Wenn darüber hinaus der Leib das unmittelbare Ausdrucksmedium einer Person ist, bildet der „Körperleib“ bzw. der „Leibkörper“ - wie die Einheit von Körper und Leib oft genannt wird $-{ }^{3}$ keine neutrale Größe, sondern ist der je eigene Leibkörper. Leiblichkeit ist nämlich dadurch gekennzeichnet, dass sie die Individualität markiert und nur durch die Erste-Person-Perspektive zur Sprache gebracht werden kann, also nicht Gegenstand wird, während Körperlichkeit umgekehrt die objektive Seite des Leibkörpers anzeigt, die andere wahrnehmen können.

Solange ein Mensch am Leben ist, kommen sein Leib und sein Körper großteils zur Deckung. Anders als im Tod, in dem deren Verknüpfung zerreißt, dissoziieren Körper und Leib während des Lebens nicht erheblich. So ist der Ort, an dem sich der Herd einer Krankheit befindet, die jemand hat, gewöhnlich auch der Ort, wo er seinen Schmerz lokalisiert. Diese „grundsätzliche Syntopie“, wie sie Thomas Fuchs nennt, (Fuchs 2017, 107; Hervorh. im Original) weist darauf hin, dass der Leibkörper eine räumli-

2 Schmitz geht davon aus, dass die „Annahme einer Identität des Leibes mit dem sinnfälligen Körper [...] nicht haltbar“ sei und „eine Ausfahrt des Leibes aus dem Körper“ seiner Auffassung nach „nicht [sic!] Erstaunliches hätte“. Er gesteht bloß die Möglichkeit eines „kausalen Einfluss[es] des Leibes auf den Körper“ zu. (Schmitz 2011, 143f.) Jens Soentgen macht dagegen geltend, dass Schmitz zwar den Leib entdeckt, aber dafür den Körper verloren habe, (Soentgen 2017, 62) und sieht darin eine „Verwechslung von Autonomie und Autarkie“ (Soentgen 2017, 64) gegeben.

3 Waldenfels unterscheidet zwischen diesen beiden Begriffen und gibt dem Ausdruck „Leibkörper“ den Vorzug, weil mit ihm die Vorherrschaft des Leibes besser zum Ausdruck komme. (Waldenfels 2000, 252) 
che Einheit ist, die nur in manchen Fällen auseinandertritt. Was für den räumlichen Aspekt gilt, kann auch für die Zeitstruktur des Verhältnisses von Leib und Körper behauptet werden. Der in der "Außeneinstellung“ der Dritte-Person-Perspektive sichtbar werdende Körper und der in der „Inneneinstellung“ der Erste-Person-Perspektive spürbare Leib sind zugleich da, sie sind „kompräsent“, wie Edmund Husserl sich ausdrückt. (Husserl 1952, 161)

Dennoch ist die Möglichkeit des Auseinandertretens von Körper und Leib ein wichtiger Hinweis darauf, dass die behauptete Einheit nicht selbstverständlich ist, sondern unter bestimmten Umständen gestört werden kann und wieder neu gewonnen werden muss. Wenn z. B. ein Organ „in seiner Gegenständlichkeit [...] aufdringlich wird“ (Coors 2019, 29 Anm. 62), weil es etwa in seiner Funktion eingeschränkt ist, kann zwar vielleicht durch einen Eingriff die Leistungsfähigkeit wiederhergestellt werden, doch ist damit noch nicht erreicht, dass die betroffene Person von sich sagen kann, sie sei wieder gesund. Es muss sich das Verhältnis zwischen Körper und Leib erst neu konsolidieren, besonders wenn es darum geht, mit einer chronischen Krankheit leben zu lernen. Auch müssen im Laufe des fortschreitenden Alters die beiden Dimensionen stets neu zur Deckung gelangen, damit der veränderten Lebensweise durch die betroffene Person entsprochen werden kann.

\section{2. „Herausverleibung“ und „Einverleibung"}

Wenn es neben der für den gewöhnlichen Lebensvollzug vorherrschenden räumlichen und zeitlichen Deckung von Körper und Leib auch Dissoziationsformen gibt, lässt sich fragen, ob und wie z. B. Gliedmaßen, wenn sie amputiert werden müssen, ihren Ausgang aus dieser Einheit finden. $\mathrm{Zu}$ gleich stellt sich das Problem, wie fremde Organe oder Gegenstände integriert werden können. Hermann Schmitz spricht von „Einleibung“ und „Ausleibung“. Für ihn ist der Schmerz ein Beispiel für „interne Einleibung“. Im Schmerz wird etwas Negatives ein Moment des Leibes selbst, sodass der Gepeinigte den Schmerz nicht direkt attackieren kann, weil er gleichsam zu seinem Leib gehört. (Schmitz 2011, 29) Demgegenüber ist die „externe Einleibung“ der Eindruck von etwas Äußerem, das sich in einem selbst manifestiert und einen nicht loslässt wie eine Melodie als „Ohrwurm“, der einen gleichsam belagert, oder zudringliche Gefühle, die man nicht beherrschen kann. (Schmitz 2011, 30f.) Mit dem Begriff „Ausleibung" meint Schmitz hingegen, dass jemand in den "Zustand des Versinkens oder der Versunkenheit" gerät, also in einer bestimmten Atmo- 
sphäre gleichsam sich selbst verliert. (Schmitz 2011, 50f.; vgl. Eberlein 2017, 40-42)

\section{1 „Herausverleibung“"}

Für das Problem, wie Organe oder Artefakte integriert oder gleichsam ausgegliedert werden können, reicht diese Terminologie nicht hin, spricht doch Schmitz in Bezug auf Schmerzen, Melodien oder Gefühle von „Halbdingen" (Schmitz 2011, 29), die für Ein- und Ausleibungen in Frage kommen. Bei Implantationen oder der Abnahme von Gliedmaßen handelt es sich nämlich nicht bloß um „Halbdinge“, sondern - wenn man so will sehr handfest um „ganze“ Dinge, obwohl Phantomglieder solchen „Halbdingen" nahekommen. Hermann Plügge spricht demgegenüber von „Wiedereinverleibung" mit Bezug auf die Integration einer Prothese bzw. vom „Herausverleiben“ mit Bezug auf ein Körperglied. (Plügge 1967, 92f.) Im Hinblick auf das „Herausverleiben“ muss zunächst nicht sofort an die Amputation gedacht werden. Es beginnt sachlich schon viel früher, wenn etwa ein schmerzendes Organ oder eine verletzte Gliedmaße aus der Sphäre des Leiblichen in jene des Körperlichen verschoben werden soll. Dabei wird versucht, den Schmerz oder das Gebrechen zu lokalisieren, also aus der Empfindung, dass „es“ schmerzt und einen als ganze Person betrifft, einen Sachverhalt zu generieren, in dem klar geworden ist, wo der Schmerz genau liegt, und möglichst auch diagnostiziert wurde, um welche Krankheit es sich handelt. Was Schmerzen bereitet, soll nicht auf mich als ganzen Menschen ausgedehnt bleiben, sondern mir so gegenübertreten, dass nicht mehr nur ich als Ganzer bzw. Ganze mein Leiden bin, sondern dass ich eine bestimmte Krankheit habe, (Waldenfels 2019, 307) die sich zu mir in Distanz setzen lässt, und sei es nur dadurch, dass die „schlimme Stelle" identifiziert oder diffuse Beschwerden diagnostiziert werden. (Plügge 1967,93 ) Es geht also darum, das Ungegenständliche des Leibes wenigstens partiell in das Gegenständliche des Körpers zu transformieren.

Dass sich der Leibkörper solchen Versuchen der Herausverleibung auch widersetzen kann, zeigt das Problem des Phantomgliedes auf. Maurice Merleau-Ponty hat es als Leib-Problem beschrieben und zu seiner Erklärung auf das Leibgedächtnis verwiesen. Im Unterschied zum Gedächtnis, dessen Inhalte in das Bewusstsein geholt werden können und das etwa im Falle einer Demenz gestört ist, meint das Leibgedächtnis die Rhythmen, Gewohnheiten und Routinen, die sich jemand im Laufe des Lebens angeeignet hat und die diesseits des Bewusstseins präsent sind oder abgerufen werden können. Im Unterschied zum „deklarativen“ oder „expliziten“ Ge- 
dächtnis des Bewusstseins ist das Leibgedächtnis ein „implizites“ Gedächtnis, das die Vergangenheit nicht vergegenwärtigt, sondern schon „latent in sich [enthält]“". (Meer 2018, 210 u. 212; Hervorh. im Original)

Es ist möglich, dass bei jemandem, dem ein Bein amputiert werden musste, das Leibbewusstsein und das Leibgedächtnis differieren. Während diese Person um das Fehlen des Körpergliedes weiß, fungiert der Leib so, als stünde das Bein noch zur Verfügung. So kann es passieren, dass der Patient beim Aufstehen aus dem Sitzen stürzt, weil in der eingeübten und unzählige Male vollzogenen Routine leiblich weiterhin davon ausgegangen wird, dass das amputierte Bein noch zur Verfügung steht. Doch der eigene Leib ist vorerst nicht mehr der unproblematische „Angelpunkt der Welt" (Merleau-Ponty 1966, 106), der es dem Amputierten erlaubte, sich ungestört in der Welt zu bewegen.

Merleau-Ponty unterscheidet zur Erklärung solcher Vorfälle zwei Dimensionen des Leibes, nämlich die "Schicht“ "des habituellen und die [Schicht] des aktuellen Leibes“ (Merleau-Ponty 1966, 107). Während die habituelle das Leibgedächtnis zu ihrer Grundlage hat, also von den in der Vergangenheit angeeigneten Gewohnheiten und Gesten geprägt ist und dementsprechend fungiert, ist die Schicht des aktuellen Leibes der faktisch disponierte Leib, der bestimmte Möglichkeiten des Weltbezugs nicht weiter gewährleistet. Kommen diese beiden Ebenen nicht mehr zur Deckung, können Gliedmaßen zu Phantomen werden, die zwar tatsächlich nicht mehr zum Leib gehören, ihm aber habituell immer noch angehören.

Um die Divergenz der beiden Schichten zu überwinden, muss die verlorene Gliedmaße „herausverleibt“, also das Leibgedächtnis so fortentwickelt werden, dass die neuen Vollzugsformen sich so im Leib sedimentieren, dass die alten Routinen als Möglichkeiten verschwinden. Der habituelle muss den aktuellen Leib einholen und die gleiche leibliche Extension wiedergewinnen. Erst dann ist das Glied aus dem leiblichen Verbund entlassen und bleibt als Körperteil zwar objektivierbar, nimmt aber im konkreten Lebensvollzug keine entscheidende Rolle mehr ein. 


\section{2 „Einverleibung“}

In manchen medizinischen Fällen geht es aber nicht nur um „Herausverleibung“, sondern auch um „Einverleibung“. Beide Vorgänge sind bei Organtransplantationen ${ }^{4}$ eng miteinander verbunden. Es muss nämlich zunächst das kranke Organ nicht nur physisch entfernt werden, sondern auch aus dem Leibganzen herausfinden, wozu kaum Zeit bleibt, ist es doch notwendig, das Spenderorgan unmittelbar nach der Entnahme des kranken zu implantieren. So tritt die „Herausverleibung“ hinter das „Einverleiben" des neuen Organs in den Eigenleib des Empfängers oder der Empfängerin zurück. Wie man weiß, sind mitunter neben den medizinischen Problemen eines solchen Eingriffs, der Abstoßungsgefahr und der Frage der Funktion des Organs im neuen Organismus auch psychische Hürden zu überwinden, insofern erst verarbeitet werden muss, dass das Organ, das im Leibkörper eines fremden Menschen, der sein Leben lassen musste, integriert war, nun das eigene Leben aufrechterhält. Darüber hinaus ist zu beachten, dass die Eingliederung in den habituellen Leib gelingen muss und dass die neuen Koordinaten des Gesamtsystems wie etwa Herzrhythmen, Immunsuppression oder Operationsfolgen innerhalb des eigenen Leibes in Balance gebracht werden.

Jean-Luc Nancy, der selbst mit einem transplantierten Herzen lebt, hat seine Situation in der Schrift Der Eindringling / L'Intrus. Das fremde Herz reflektiert. Nicht nur durch das Herz, das 20 Jahre jünger ist als er selbst, sondern auch durch die schweren Folgeerkrankungen, unter denen er leidet, sieht er die eigene Identität gestört. Nancy sieht aufgrund der Konsequenzen, die die Unterdrückung der Abwehrkräfte nach sich zieht, indem sie zwar eine Abstoßungsreaktion verhindert, aber andere Krankheiten hochkommen lässt, sich selbst entfremdet. (Feichtinger 2019, 204) Er resümiert diese seine Lage in Hinblick auf sein transplantiertes Herz nüchtern: „Daß ich mir selber fremd werde, bringt mich jedoch nicht dem Eindringling näher." (Nancy 2000, 35) Er meint damit, dass seine Identität dauerhaft gestört bleibt, sodass er auch in Bezug auf die notwendigen Folgebehandlungen konstatieren muss: „Man erkennt sich nicht wieder.“ (Nancy 2000, 43)

Der Text Nancys über sein fremdes Herz zeigt, wie schwierig sich die Aneignung eines fremden Organs gestaltet und dass eine völlige „Einverleibung" praktisch unmöglich erscheint, obwohl das transplantierte Herz

4 Einen historischen Überblick über die Entwicklung der Transplantationsmedizin bietet von Engelhardt (2015). 
sein Weiterleben möglich macht und die Integration des fremden Organs in seinen Leib zumindest teilweise gelungen ist. Wie aber stellt sich die Lage dar, wenn nicht ein Organ eines fremden Menschen, sondern ein Artefakt einverleibt werden soll? Merleau-Ponty verweist in diesem Zusammenhang auf das Beispiel eines Blinden. Sein Stock ist nicht mehr nur als ein einfaches Werkzeug, das noch „für sich selbst“ als Gegenstand wahrgenommen würde, anzusehen. Vielmehr gilt: Der Blindenstock „vergrößert Umfänglichkeit und Reichweite des Berührens“ und ist „zu einem Analogon des Blicks geworden“. (Merleau-Ponty 1966, 173) Der Blinde hat den Stock nämlich so angeeignet, dass er sogar den verloren gegangenen Sehsinn weitgehend kompensiert, der Stock ist auf diese Weise gleichsam zur verlängerten Hand mutiert. Ähnliche Beispiele, auf die ebenfalls MerleauPonty hinweist, sind das durch Gewohnheit erlernte, aber vorbewusste genaue Abschätzen von Abständen für den Hut, den man auf dem Kopf trägt, oder das Zusammenzucken einer Person, die ein Auto lenkt, das Gefahr läuft, bei einer engen Durchfahrt an der Mauer anzufahren. Es scheint, als sei das Auto so inkorporiert worden, als markierten die Umrisse des Fahrzeugs die eigenen Körpergrenzen. (Merleau-Ponty 1966, 172f.)

Spektakulärer, weil ungewohnt, erscheinen Experimente, die „Einverleibungen" künstlich provozieren. So ist es möglich zu insinuieren, dass man anstelle einer der beiden eigenen Hände eine Gummihand in den eigenen Leib integriert habe. Bei diesen Versuchen sitzt eine Person an einem Tisch, auf den sie beide Arme legt. Allerdings ist nur der eine für sie selbst sichtbar, den anderen legt sie hinter einen kleinen Schirm, der ihn verdeckt und ihren Blicken entzieht. Nun wird gleichsam als Attrappe ein Gummiarm vor die Versuchsperson auf den Tisch positioniert, und die Versuchsleitung streichelt im gleichen Rhythmus zugleich die Handoberfläche des verdeckten Arms und die sichtbare Handoberfläche des Gummiarms. Nach einiger Zeit spürt die Versuchsperson den fremden Arm als eigenen und schreit oder zuckt zusammen, wenn man den Gummiarm etwa mit einem Hammer „verletzt“. (Botvinick/Cohen 1998; Fuchs 2017, 99 Anm. 9)

Bemerkenswert ist, dass mittlerweile ein analoges Experiment auch mit Handys funktioniert hat, nicht jedoch Versuche, bei denen man den Gummiarm durch andere Gegenstände ersetzt hat. War es beim Gummiarm noch die Ähnlichkeit mit einem eigenen Körperteil, so liegt die Täuschungsmöglichkeit mit dem Handy offenbar daran, dass der Gebrauch tragbarer Telefone - ähnlich wie der Umgang des Blinden mit seinem Stock - bereits so vertraut ist, dass sie gleichsam wie die Erweiterung der eigenen Hand aufgefasst werden. Freilich muss der Gesichtssinn vermitteln, denn ohne dass die Versuchsperson beständig auf das dargebotene Ar- 
tefakt blickt, funktioniert das Experiment nicht. Zudem muss man den Gegenstand gewohnt sein, denn selbst bei einer Computer-Maus oder dem Holz-Imitat eines Smartphones ließ sich der Effekt nicht erzeugen. (Liepelt/Dolk/Hommel 2017)

Solche Versuche zeigen neuerlich zweierlei: zum einen, dass die Extension von Körper und Leib nicht denselben Umfang haben muss, und zum anderen, dass die Einverleibung von Artefakten möglich ist, selbst wenn die Angewöhnung nicht lange dauert. Anders als bei der Transplantation wird hier nicht ein lebenswichtiges Organ dauerhaft ersetzt, dessen „Herausverleibung“ lebensbedrohlich wäre. Vielmehr wird ein zusätzliches Teil zeitlich beschränkt in das „Körperschema“ (Merleau-Ponty 1966, 123) übernommen und in den eigenen Leib integriert. Seltsamerweise empfindet die Versuchsperson das Smartphone ja selbst als eigene Gliedmaße. Freilich ist es nicht möglich, das eigene Handy dauerhaft zu integrieren, da dafür Propriozeption vorausgesetzt werden müsste. Des Weiteren ist für eine dauerhafte Aneignung unumgänglich, dass sich jemand das Artefakt nicht nur leiblich aneignet, sondern dass es zuvor auch Teil des eigenen Körpers geworden ist. So geht im Versuch mit dem Smartphone nach den ersten Bewegungen die Empfindung wieder verloren, weil das Smartphone liegen bleibt und eben nicht in der eigenen Hand getragen wird, sodass man mit dem Handy auch die eigene Hand spürte. Leibliche Integration ohne körperliches Fundament bleibt also ephemer.

So ist es das Bestreben, technisch hergestellte Prothesen zu entwickeln, die es ermöglichen, sie vor der „Einverleibung“ zu inkorporieren, also mit dem Körper zu verknüpfen. Das heißt, dass Prothesen so mit dem Körper verbunden werden sollen, dass sie Nervenimpulse nach außen übertragen und umgekehrt Außenreize so nach innen vermitteln, dass diese auch empfunden werden. Auf der einen Seite sollen die einer Willensregung entspringenden Impulse aus dem Gehirn direkt in die Prothese übertragen werden, damit Betroffene mit der Prothese wie mit einem eigenen Körperglied operieren können. Andererseits soll der Glied-Ersatz in der Person direkt eine Empfindung auslösen. So hat z. B. das Centre Suisse d'Electronique et de Microtechnique CSEM mit zehn Partnern im Rahmen des auch von der EU unterstützten Projekts DeTOP jüngst eine Handprothese entwickelt, in der die Elektroden direkt in die Nerven der Muskeln im Stumpf eingepflanzt werden. Diese Technik ermöglicht nicht nur anspruchsvolle Bewegungen durch die Kunsthand, sondern auch das Tasten mittels der Prothese. Dafür wird die Schnittstelle in den Knochen integriert, sodass eine direkte elektronische Verbindung zwischen der Prothese und den Elektroden in den Nervenenden möglich ist. (Kombination 2019) Sollen Prothesen dieser Machart „einverleibt“ werden, so ist zu hoffen, dass durch die 
Praxis des Lebensvollzugs ein Leibgedächtnis aufgebaut wird, das es ermöglicht, sie in der Anwendung immer mehr ins Leibschema zu integrieren.

\section{Aspekte körperlicher und leiblicher Aneignung}

Die angeführten Beispiele für die Integration von Artefakten und die damit einhergehenden Probleme zeigen, dass es mit der Angliederung oder Einpflanzung von Hightech-Gegenständen allein nicht getan ist, sollen diese so einverleibt werden, dass sie im Lebens- und Selbstvollzug integriert sind und nicht immer wieder negative Aufmerksamkeit erregen. Trotz aller Abstufungen und unterschiedlichen individuellen Problemlagen lässt sich sagen, dass eine Grundvoraussetzung für dauerhafte Einverleibung die vorhergehende funktionale und physiologische Zusammenführung ist, also die körperliche Integration. Erst dann kann die allmähliche Integration in den Leib erfolgen, sodass Betroffene sich so weit mit den implantierten Organen oder Gliedmaßen identifizieren, dass sie sie als Teil ihrer selbst empfinden. Hüft- oder Knieprothesen werden besonders dann als integriert erlebt, wenn Bewegungen wie Gehen oder Laufen wieder schmerzlos möglich sind. Das setzt aber voraus, dass ein entsprechendes Leibgedächtnis über entsprechende Rehabilitation aufgebaut worden ist. Schwieriger ist die Situation freilich, wenn ganze Gliedmaßen ersetzt werden, möglicherweise technische Probleme auftreten oder Gewöhnungsphasen lange dauern.

So lässt sich als erster zentraler Aspekt der „Einverleibung“ das Verhältnis von innen und außen namhaft machen. Diese Relation erhält im Zusammenhang der Integration von Organen oder Prothesen aber eine seltsame Verdoppelung, sofern man nicht übersieht, dass der eigene Leibkörper sowohl das Moment enthält, dass man mit ihm identisch ist, als auch das Moment, dass man ihm gegenübertreten kann. So ist das erste Außen die Tatsache, dass Spenderorgane, künstliche Gelenke oder Prothesen einem selbst fremd sind und dem eigenen Körper erst eingepflanzt oder mit ihm verbunden werden müssen. Gelingt dies, ist zunächst bloß die Integration in den eigenen Körper erfolgt. Das erste Innen ist also körperlicher Natur. Denn das integrierte Organ oder Artefakt gehört dann zwar dem eigenen Körper an, bleibt aber ein Gegenüber, mit dem noch keine Identifikation von Seiten des Ichs erfolgt ist. In dieser Situation ist etwa eine Prothese zwar Teil des eigenen Körpers geworden, aber der oder die Betroffene würde nicht sagen, diese Prothese auf die gleiche Weise zu sein, wie er oder sie sagen könnte, das eigene Auge zu sein. Der körperliche Innenraum ist 
noch kein leiblicher, oder anders gesagt: Das körperliche Innen bleibt zunächst noch ein leibliches Außen.

Erst wenn das Organ, die Prothese oder die künstliche Gliedmaße darüber hinaus in das Leibschema Eingang findet und die damit verbundenen Vorgänge und Abläufe als vorbewusste Routinen ins Leibgedächtnis aufgenommen worden sind, kann das körperliche Innen auch zu einem leiblichen werden. Doch sind dies zwei zu unterscheidende Vorgänge, die sich etwa bei der Implantation eines Hüftgelenks beobachten lassen. Das körperliche Innen ist dann erreicht, wenn die Operation gelungen ist und das Gelenk wieder funktioniert. Von einem leiblichen Innen kann man aber erst dann sprechen, wenn der Aufbau des Leibgedächtnisses durch eine Rehabilitation und durch Bewegungsübungen so weit gediehen ist, dass die betroffene Person wieder so gut gehen kann, dass das operierte Bein unter die gewöhnliche und alltägliche Aufmerksamkeitsschwelle gefallen ist. Das künstliche Gelenk wird erst dann so empfunden, als sei es das eigene, wenn gehen zu können wieder alltäglich geworden ist. Erst wenn dieser Zustand eintritt, ist das körperliche Innen, das zugleich noch ein leibliches Außen gewesen ist, zu einem auch leiblichen Innen geworden.

Mit dieser Verdopplung von innen und außen ist als zweiter Aspekt eine Schwierigkeit angezeigt, ausformuliert in der phantastisch anmutenden Frage, wie viel an Körperteilen durch Artefakte ausgetauscht werden kann, ohne dass damit die Identität der betroffenen Person wesentlich verändert wird bzw. der Mensch stirbt. Wesentlich für die Beantwortung dieser Frage ist zweifellos der Grad der leiblichen und nicht nur körperlichen Integration schon implantierter Ersatzglieder oder Kunstorgane. Nur wenn solche ganz Leib geworden sind oder - allgemeiner gesprochen überhaupt ganz Leib werden können, macht die Science-FictionVorstellung Sinn, dass am Ende zunehmender Ersetzung schließlich ein rein künstlich hergestellter Mensch übrig bliebe, der selbst von sich sagen könnte, all diese Erzeugnisse seien sein Leib.

Analog dazu lässt sich fragen, ob ein humanoider Roboter in Zukunft einmal nicht bloß Körper - freilich künstlich hergestellter Körper -, sondern zugleich Leib sei, also von sich in der Erste-Person-Perspektive sagen könnte, er sei selbst die Schaltkreise, Halbleiter und einzelnen Maschinenteile, die in ihm miteinander verbunden sind. Ein solcher humanoider Roboter müsste, sollte er als leibliches Wesen gelten können, nicht nur ein Bewusstsein haben, sondern auch ein Innen und ein Außen kennen, sodass ihm der Tausch eines Teiles seiner selbst Schmerzen verursachen könnte. Wäre dies nicht der Fall, ließe sich schwerlich sagen, dass es sich um eine leibliche Existenz handelt. Es gäbe nämlich nur ein Außen ohne Innen. 
Umgekehrt könnte man analog zu Neurophilosophen wie Thomas Metzinger im Menschen und weiter in einem Roboter einen „Ego Tunnel“ ansetzen, aus dem es kein Entkommen gibt, weil die Innenwelt für die Außenwelt gehalten wird und man meint, das Gehirn konstruiere eine bloß vermeintlich existierende Außenwelt. Wieder verschwindet die Polarität zwischen innen und außen und wird auf ein bloßes Innen reduziert. Hier würde nicht nur die Körperlichkeit, sondern letztlich auch die Leiblichkeit verschwinden, da es nur mehr das Gehirn als Rechenzentrum gibt, das einem eine Welt vorgaukelt. Man empfindet nach dieser Auffassung die Welt nicht mehr, sondern befindet sich bloß in der „Illusion, in unmittelbarem Kontakt zur Welt zu stehen“ (Metzinger 2009, 70). Ein Leibkörper hingegen ist undenkbar ohne das Spannungsverhältnis von innen und außen, das sich in der Polarität von Körper und Leib zeigt. Phänomenal gesehen, widersprechen also sowohl Physikalismus als auch Konstruktivismus der Erfahrung, Leib zu sein und einen Körper zu haben. Darüber hinaus kann das Problem der „Herausverleibung“ bzw. der „Einverleibung“ nicht erklärt werden, sobald die Polarität von innen und außen nivelliert wird. Das sich immer neu manifestierende Verhältnis eines doppelten Innen und eines doppelten Außen verschwindet nämlich. Ohne Leib kann es naturgemäß keine leibliche Aneignung geben. Dann bleibt die Maschine eine tote Maschine, denn Lebendiges, zumal der Mensch, zeichnet sich gerade dadurch aus, dass es sowohl den "organismisch-körperlichen" wie auch den „subjektiv-leiblichen Aspekt“ zugleich in sich enthält. (Fuchs 2017, 100f.)

Ein dritter Gesichtspunkt, den es zu erwähnen gilt, ist das Verhältnis von eigen und fremd, das mit der Relation von innen und außen analog gesetzt werden kann, aber zusätzlich das Moment eigener Identität beibringt. Während der erwähnte Physikalismus das Identitätsproblem eines Menschen mit einem fremden Körperteil insofern wegerklärt, als er den eigenen Leib in einen fremden Körper auflöst, verkürzt der Konstruktivismus, der einen „Ego Tunnel“ ansetzt, das Identitätsproblem so, dass es nur mehr Eigenes, aber nichts mehr Fremdes gibt. Nicht einmal die eigene Umwelt hat demnach etwas Fremdes an sich, da auch sie bloß ein vom Gehirn produziertes Bild ist. Die Analyse des Verhältnisses von Körper und Leib hat hingegen darauf aufmerksam gemacht, dass der Leibkörper - besonders bei Problemen der „Herausverleibung“ und der „Einverleibung“ Ort des Verlustes und der Gewinnung von Gleichheit ist. Das zeigen nicht nur die Schwierigkeiten, sich mit einem implantierten Organ identifizieren zu können, sondern auch Belastungen bei der Einpflanzung künstlicher Prothesen. Identität muss generell je neu errungen werden und bildet für Menschen mit fremden Organen oder Artefakten im eigenen Leib ein 
noch größeres Problem als für Menschen, die ein solches Los nicht tragen müssen.

Doch ist das Problem der ständig neu zu gewinnenden Einheit mit sich selbst - neben seiner psychischen Seite - immer auch eine leib-körperliche Aufgabe. Das liegt daran, dass der Leibkörper in jedem Fall immer schon Fremdes in sich enthält. Denn die Identität zwischen Leib und Körper bricht immer wieder auf, wenn etwa der Selbstausdruck nicht gelingt, weil man sich sprachlich verhaspelt, oder wenn man stolpert, einem etwas aus der Hand fällt oder wenn man Fehler macht, weil man ermüdet. (Waldenfels 2019, 307) Der Leibkörper hat immer wieder die Tendenz, einem von sich aus partiell fremd zu werden. So gilt es, die eigene leib-körperliche Identität beständig wiederzugewinnen, bis hin zur Identifizierung des eigenen Leibkörpers am Morgen mit jenem, den man beim Einschlafen gleichsam zurücklassen musste. (König 2019) Nur weil der Leibkörper immer schon in der Spannung von Eigenem und Fremdem steht, ist eine Implantation, also der Transfer von etwas Fremdem in das Eigene, überhaupt erst möglich - freilich um den Preis, dass mit ihm diese Spannung nicht verschwindet, sondern sich verstärkt, weil man als leib-körperliches Selbst Identität mit Fremdem erst gewinnen muss und die Gefahr der anschließenden Entfremdung nicht gebannt werden kann.

\section{Schluss}

Die Aneignung von Organen, künstlichen Gelenken oder Prothesen - so haben die durchgeführten Reflexionen gezeigt - kann weder bloß als technischer Prozess der Ersetzung oder des Austausches von Ersatzteilen verstanden werden noch als die Eingliederung in ein physiologisches oder biochemisches System. Vielmehr ist sichtbar geworden, dass Implantationen ein körperlicher und zugleich ein leiblicher Vorgang sind, bei dem einerseits ein doppeltes Außen zu überwinden und andererseits ein Prozess der Identifizierung zu leisten ist. Dies verlangt neben mentaler Anverwandlung vor allem eine „Einverleibung“. Eine solche ist aber nicht nur ein Geschehen, das durch externe Zugriffe in Gang gebracht wird, sondern ein subjektiver Vorgang, der sich weder allein aktiv noch bloß passiv ereignet, also nicht durch die alleinige Anstrengung des Bewusstseins gelingen kann. Vielmehr muss eine solche Aneignung leiblich und darüber hinaus als das Zugleich von aktivem Bemühen und passivem Geschehen erfolgen. Damit liegt sie aber nicht allein in der Verfügung der betroffenen Person und ist nicht einfach herstellbar. Denn ohne dass Implantate wie von 
selbst im leiblichen Selbstvollzug aufgehen und sich im Leibgedächtnis sedimentieren, wird die Aneignung von Artefakten nicht erfolgreich sein.

\section{Literatur}

Botvinick, Matthew/Cohen, Jonathan: Rubber hands 'feel' touch that eyes see, in: Nature 391 (1998) 756.

Coors, Michael: Narrative des guten Sterbens. Zur Normativität narrativer Schemata in der ethischen Diskussion über das Lebensende, in: Praxis Palliative Care 42 (2019) 21-32.

Eberlein, Undine: Aspekte leiblicher Intersubjektivität, in: Volke, Stefan/Kluck, Steffen (Hg.): Körperskandale. Zum Konzept der gespürten Leiblichkeit, Freiburg/Br.: Alber 2017 (= Neue Phänomenologie 27), 39-57.

Engelhardt, Dietrich von: Organtransplantation und Leiblichkeit. Kulturhistorischer Kontext - klinische Herausforderungen, in: Kick, Hermes Andreas/ Schmitt, Wolfram (Hg.): Leib und Leiblichkeit als Krisenfeld in Psychopathologie, Philosophie, Theologie und Kunst. Ansätze zu einer interdisziplinären Anthropologie von Entsprechen und Verantworten. Alfred Kraus zum 80. Geburtstag, Münster: Lit 2015 (= Affekt - Emotion - Ethik 15), 31-49.

Feichtinger, Daniela: Wo sind die Körper? Körper und Ort bei Jean-Luc Nancy und in ausgewählten Exponaten der Ausstellung, in: Del Guercio, Andrea B./Guanzini, Isabella/Ruckenbauer, Hans-Walter/Terracciano, Ida (Hg.): Kunst heilt Medizin. Interdisziplinäre Untersuchungen zu vulnerabler Körperlichkeit. Unter Mitarbeit von Isabella Bruckner, Innsbruck: Tyrolia 2019 (= Theologie im kulturellen Dialog 34), 195-208.

Fuchs, Thomas: Zwischen Leib und Körper, in: Hähnel, Martin/Knaup, Marcus (Hg.): Leib und Leben. Perspektiven für eine neue Kultur der Körperlichkeit, Darmstadt: Wissenschaftliche Buchgesellschaft 2013, 82-93.

Fuchs, Thomas: Die Koextension von Leib und Körper. Von Phantomgliedern, Gummihänden und anderen Rätseln, in: Volke, Stefan/Kluck, Steffen (Hg.): Körperskandale. Zum Konzept der gespürten Leiblichkeit, Freiburg/Br.: Alber 2017 (= Neue Phänomenologie 27), 96-115.

Husserl, Edmund: Ideen zu einer reinen Phänomenologie und phänomenologischen Philosophie. 2. Phänomenologische Untersuchungen zur Konstitution. Hg. v. Marly Biemel, Haag: Martinus Nijhoff 1952 (= Husserliana 4).

König, Anna: Erwachen. Ein leibzeitliches Übergangsphänomen, in: Esterbauer, Reinhold/Paletta, Andrea/Meer, Julia (Hg.): Der Leib und seine Zeit. Temporale Prozesse des Körpers und deren Dysregulationen im Burnout und bei anderen Leiberfahrungen, Freiburg/Br.: Alber 2019, 167-187.

Kombination von Roboter-, Sensor- und Schnittstellen-Technik. Künstliche Gliedmaßen, in: https://www.medizin-und-elektronik.de/sonstige/artikel/162616/ [20.09.2019]. 
Liepelt, Roman/Dolk, Thomas/Hommel, Bernhard: Self-perception beyond the body: the role of past agency, in: Psychological Research 81/3 (2017) 549-559.

Meer, Julia: Die Erinnerung des Leibes. Zur Relevanz und Funktion von Leibzeit bei Alzheimer-Demenz, in: Zeitschrift für praktische Philosophie 5/1 (2018) 207-230.

Merleau-Ponty, Maurice: Phänomenologie der Wahrnehmung. Aus d. Franz. übers. u. eingeführt durch eine Vorrede v. Rudolf Boehm, Berlin: de Gruyter 1966 (= Phänomenologisch-Psychologische Forschungen 7).

Metzinger, Thomas: Der Ego-Tunnel. Eine neue Philosophie des Selbst: Von der Hirnforschung zur Bewusstseinsethik, Berlin: Berlin-Verlag 2009.

Nancy, Jean-Luc: Der Eindringling/L'Intrus, in: Ders.: Der Eindringling / L'Intrus. Das fremde Herz, Berlin: Merve 2000 (= Internationaler Merve Diskurs 226), 651.

Plessner, Helmuth: Lachen und Weinen. Eine Untersuchung der Grenzen menschlichen Verhaltens (1941), in: Ders.: Gesammelte Schriften. 7. Ausdruck und menschliche Natur, Frankfurt/M.: Suhrkamp 1982, 201-387.

Plügge, Herbert: Der Mensch und sein Leib, Tübingen: Niemeyer 1967 (= Forschungen zur Pädagogik und Anthropologie 9).

Schmitz, Hermann: Der Leib, Berlin: de Gruyter 2011 (= Grundthemen Philosophie).

Soentgen, Jens: Probleme des Schmitz'schen Leibkonzeptes. Ein Kommentar, in: Volke, Stefan/Kluck, Steffen (Hg.): Körperskandale. Zum Konzept der gespürten Leiblichkeit, Freiburg/Br.: Alber 2017 (= Neue Phänomenologie 27).

Waldenfels, Bernhard: Das leibliche Selbst. Vorlesungen zur Phänomenologie des Leibes. Hg. v. Regula Giuliani, Frankfurt/M.: Suhrkamp 2000 (= stw 1472).

Waldenfels, Bernhard: Erfahrung, die zur Sprache drängt. Studien zur Psychoanalyse und Psychotherapie aus phänomenologischer Sicht, Berlin: Suhrkamp 2019 (= stw 2283). 
